\title{
Postoperative Severe Headache Following Cervical Posterior Surgical Fixation from C2 Distally
}

\author{
Ryoji Tauchi ${ }^{1}$, Sang-Hun Lee ${ }^{2}$, Jin-Young Kim ${ }^{3}$, Yong-Chan $\mathrm{Kim}^{4}$, Colleen Peters ${ }^{3}$, \\ Shiro Imagama ${ }^{1}$, Naoki Ishiguro ${ }^{1}$, Jacob Buchowski ${ }^{3}$, K. Daniel Riew ${ }^{5}$ \\ ${ }^{1}$ Department of Orthopaedic Surgery, Nagoya University Graduate School of Medicine, Nagoya, Japan \\ ${ }^{2}$ Department of Orthopaedic Surgery, Kyung-Hee University School of Medicine, Seoul, Korea \\ ${ }^{3}$ Department of Orthopaedic Surgery, Washington University in St. Louis, St. Louis, MO, USA \\ ${ }^{4}$ Department of Orthopaedic Surgery, Hallym University Sacred Heart Hospital, Seoul, Korea \\ ${ }^{5}$ Department of Orthopaedic Surgery, Columbia University, New York, NY, USA
}

Study Design: Retrospective study.

Purpose: To identify the prevalence of severe headache occurring after cervical posterior surgical fixation (PSF) and to evaluate the clinical and radiological findings associated with severe headache after surgery.

Overview of Literature: Several studies have reported on the axial pain after cervical surgery. However, to our knowledge, the incidence of severe headache after cervical PSF has not been elucidated.

Methods: The medical records and radiological assessment of patients who underwent surgical treatment from August 2002 to May 2012 were reviewed to identify the prevalence and risk factors for severe headaches occurring following PSF from C2 distally. Neck disability index scores (NDI) (the item for neck pain), the type of C2 screw, number of cervical fused levels (1-6), and smoking habit were calculated preoperatively and postoperatively. In addition, radiological parameters (T1 slope angle, C1/2 angle, C2-7 Cobb angle, C2-7 sagittal vertical axis and C1-implant distance) were assessed for all patients. Severe headache was defined as a high NDI headache score (>4 out of 5 ).

Results: Eighty-two patients met the inclusion criteria. The mean age was 59.2 years (range, 21-78 years), and the mean number of fused levels was 5.1. The mean follow-up period was 2.9 years (range, 1-10.9 years). While only one severe headache occurred de novo postoperatively in a patient in the C3 or C4 distally group (total 30 patients, average age of 50.2 years), 11 patients in the C2 distally group $(p=0.04)$ had severe headache occur postoperatively. The radiological parameters were not significantly different between the postoperative milder headache and severe headache (SH) groups. The SH group had a significantly higher preoperative NDI score (neck pain) $(p<0.01)$.

Conclusions: Newly occurring severe headaches can occur in 18\% of patients after PSF from C2 distally. The patients with newly occurring severe headaches had significantly higher preoperative NDI score (neck pain).

Keywords: Posterior surgical fixation; Cervical; Headache; Surgery

Received Oct 15, 2015; Revised Nov 22, 2015; Accepted Nov 24, 2015

Corresponding author: Ryoji Tauchi

Department of Orthopaedic Surgery, Nagoya University Graduate School of Medicine,

65 Tsurumai, Showa, Nagoya, 4668550 Japan

Tel: +81 52744 1908, Fax: +81 52744 2260, E-mail: ryojitauchi@gmail.com 


\section{Introduction}

Neck pain originates from paraspinal tissue, but chronic neck pain with concomitant headache may have underlying spine pathology, such as cervical degenerative disc disease [1]. Following cervical posterior surgical fixation (PSF) from C2 distally (PSF from C2 distally), we found that some patients began experiencing severe headache, which was not a symptom previously. Other cases had continued headache symptoms or the headache was aggravated after surgery. The factors surrounding the incidence of severe headache after cervical posterior fixation are not clear, and it is unknown whether PSF from C2 distally would be an effective procedure for treating severe headache. In addition, the actual reason for newly occurring severe headache after PSF from C2 distally has been unclear.

The authors hypothesized possible reasons why a severe headache may occur after PSF from C2 distally: (1) the implant may influence the bony structure, (2) severe muscle tension may occur due to the severe forward bending alignment of the head, and (3) C1-C2 hyperlordosis can result in $\mathrm{C} 2$ foraminal stenosis when attempting to maintain horizontal gaze. To the best of our knowledge, this is the first study of severe headache occurring after PSF from C2 distally. Its purpose is to identify the prevalence of severe headache occurring after PSF from C2 distally and to evaluate the clinical and radiological findings associated with severe headache after surgery.

\section{Materials and Methods}

\section{Patient selection and clinical investigation}

This study was approved by the Institutional Review Board at Washington University School of Medicine. We performed a retrospective review on both prospectively and retrospectively collected data for this study. The medical records and radiological assessment of patients who underwent surgical treatment from 2002 to 2012 were reviewed to identify the prevalence of and risk factors for severe headache occurring after PSF from C2 distally. Patients with less than one year follow-up, incomplete clinical data and questionnaire or occipito-cervical spontaneous fusion by ankylosing spondylitis were excluded. We also excluded patients from statistical analysis who had postoperative severe headache due to penetration of the $\mathrm{C} 2$ screw into the cortex of the $\mathrm{C} 2$ pars area directly irritating the $\mathrm{C} 2$ nerve root.

Neck disability index (NDI) scores, the C2 screw type (pedicle screw, pars screw, laminar screw, transarticular screw or combination), cervical fused levels (1-6), and smoking habit were recorded preoperatively and postoperatively. We used the NDI (headache and neck pain items) to assess patients' headache and neck pain. The NDI is a reliable, valid and sensitive instrument for selfreporting measures [2]. It includes a headache severity index, thus, we graded preoperative and postoperative headache as milder headache (grade $0-3$ ) or severe headache (SH) (grade 4 or 5). We also compared $\mathrm{SH}$ which first occurred after PSF from C2 distally with $\mathrm{SH}$ that occurred after PSF from C3 or C4 distally (30 patients), for which we adopted the same inclusion criteria (Fig. 1).

\section{Radiologic evaluation}

The following radiological spinal parameters were assessed: (1) T1 slope angle (the angle between a horizontal line and the upper end plate of T1), (2) C1-C2 lordosis angle (the angle of intersection between a line connecting the anterior tubercle to the posterior margin of the $\mathrm{C} 1$ spinous process and a line parallel to the inferior end plate of C2), (3) C2-7 Cobb angle, (4) C2-7 sagittal vertical axis (SVA: the distance between the plumb line dropped from the center of $\mathrm{C} 2$ and the posterior superior corner of C7) [3] and (5) C1-implant distance (the distance from the most proximal implant to $\mathrm{C} 1 \mathrm{arch}$ on neutral lateral cervical X-ray) (Fig. 2).

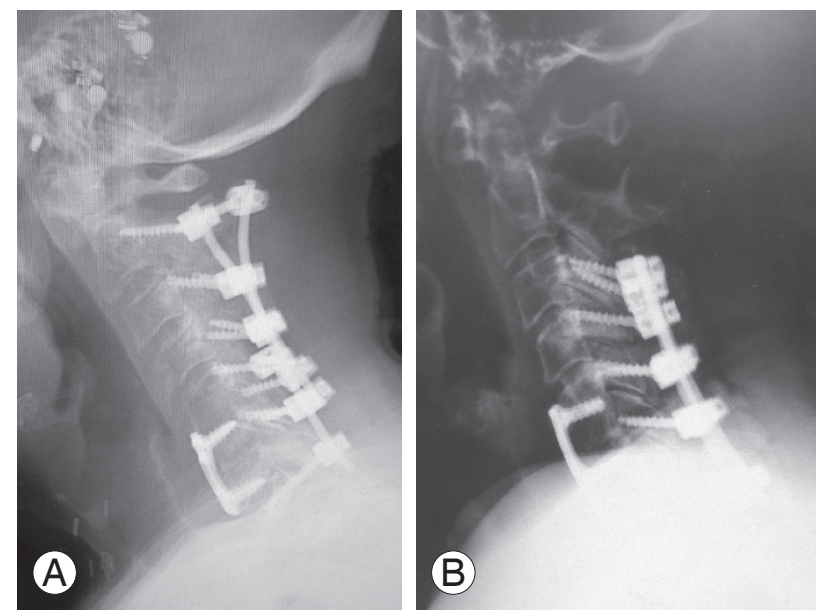

Fig. 1. (A) Posterior surgical fixation C2 distally. (B) Posterior surgical fixation C3 distally. 

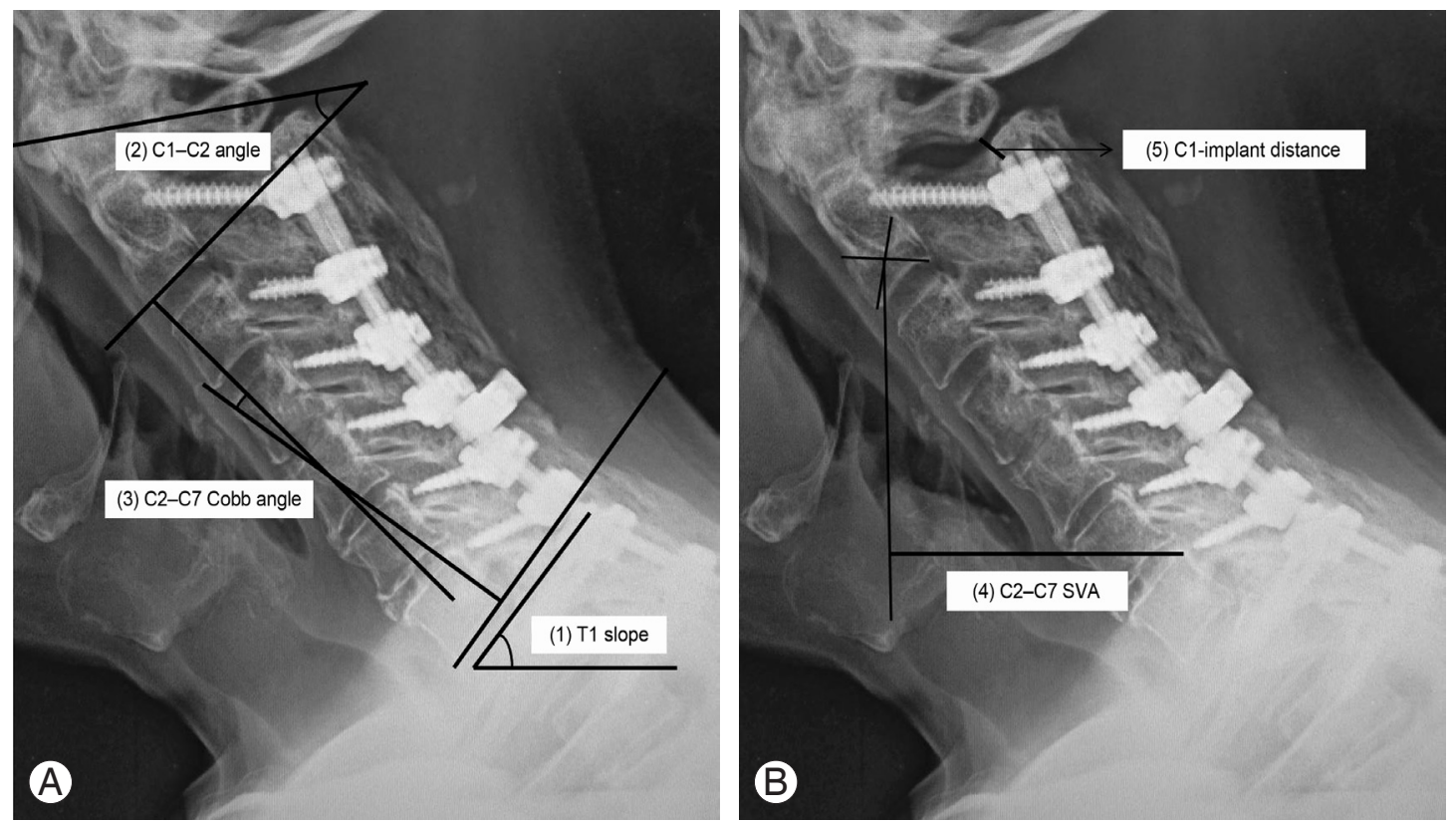

Fig. 2. (A) Radiological parameters. (1) T1 slope angle: the angle between a horizontal line and the upper end plate of T1. (2) C1-C2 lordosis angle: the angle of intersection between a line connecting the anterior tubercle to the posterior margin of the C1 spinous process and a line parallel to the inferior end plate of C2. (3) C2-7 Cobb angle. (B) Radiological parameters. (4) C2-7 sagittal vertical axis (SVA): the distance between the plumb line dropped from the center of C2 and the posterior superior corner of C7. (5) C1-implant distance: the distance from the most proximal implant to C1 arch on neutral lateral cervical X-ray.

\section{Statistical analysis}

Data were analyzed using SPSS ver. 20.0 (IBM Co., Somers, NY, USA). We used Mann-Whitney U-tests for non-normally-distributed variables and chi-square tests for categorical variables. The threshold for significance was a $p$-value of $<0.05$.

\section{Results}

\section{Severe headache after PSF from C2 distally compared with PSF from C3 or C4 distally}

Eighty-two patients met the inclusion criteria for patients receiving PSF from C2 distally. There were 44 males and 38 females, with an average age of 59.2 years (range, 21-78 years). The mean follow-up periods were 2.9 years (range, 1-10.9 years). The mean number of cervical fused levels was 5.1 (1-6 levels). On the other hand, in the PSF C3 or C4 distally group, 30 patients (average age of 50.2 years) met the inclusion criteria (Table 1). While only one patient in the PSF from C3 or C4 distally group had severe headache newly occur after surgery, severe headache newly occurred postoperatively in 11 patients in the PSF from C2 distally group $(p=0.04)$.

\section{Clinical and radiological findings in $\mathbf{6 0}$ patients who demonstrated milder headache preoperatively}

Among all PSF from C2 distally patients, 60 patients demonstrated preoperative milder headache. Of these 60 patients, 49 patients continued to have milder NDI headache scores (new headache-group) postoperatively, while 11 patients had newly occurring severe headache (new headache+group) postoperatively. The radiological parameters (T1 slope angle, $\mathrm{C} 1-\mathrm{C} 2$ angle, $\mathrm{C} 2-7 \mathrm{Cobb}$ angle, C2-7 SVA and C1 implant distance) were not significantly different from both groups. The new headache+group had significantly higher score for preoperative NDI for neck pain $(p<0.01)$ (Table 2).

\section{Discussion}

No previous studies have evaluated severe headache after cervical PSF. In the present study, we found that a newly occurring severe headache after cervical PSF was more frequent in C2 distally fusions than in $\mathrm{C} 3$ or $\mathrm{C} 4$ distally 
Table 1. Severe headache after PSF from C2 distally compared with PSF from C3 or C4 distally

\begin{tabular}{|c|c|c|c|}
\hline Variable & C2 distally (n=82) & C3 or C4 distally $(n=30)$ & $p$-value \\
\hline Age (yr) & 59.2 & 50.2 & $<0.001$ \\
\hline Sex (male/female) & $44 / 38$ & $15 / 15$ & 0.731 \\
\hline Fused level & 5.1 & 3.9 & $<0.001$ \\
\hline \multicolumn{4}{|l|}{ Post NDI (headache) } \\
\hline Grade 0 & 17 & 4 & \\
\hline Grade 1 & 18 & 9 & \\
\hline Grade 2 & 15 & 10 & \\
\hline Grade 3 & 12 & 4 & \\
\hline Grade 4 & 10 & 1 & \\
\hline Grade 5 & 10 & 2 & \\
\hline Average & 2.1 & 1.8 & 0.538 \\
\hline New incidence of severe headache & $11^{\text {a) }}$ & 1 & 0.043 \\
\hline
\end{tabular}

PSF, posterior surgical fixation; NDI, neck disability index. a) $p<0.05$.

Table 2. Clinical and radiological findings in 60 patients who demonstrated milder headache preoperatively

\begin{tabular}{|c|c|c|c|}
\hline Variable & New headache $-(n=49)$ & New headache $+(n=11)$ & $p$-value \\
\hline Age (yr) & 60.9 & 56.2 & 0.062 \\
\hline Sex (male/female) & $28 / 21$ & $3 / 8$ & 0.055 \\
\hline Preoperative NDI (neck pain) & 1.6 & 3.0 & 0.001 \\
\hline Fused levels & 5.2 & 5.1 & 0.775 \\
\hline Smoking (n [\%]) & $20(36)$ & $5(44)$ & 0.567 \\
\hline T1 slope angle (degrees) & 36.8 & 32.1 & 0.184 \\
\hline C1-2 angle (degrees) & 34.6 & 34.1 & 0.695 \\
\hline C2-7 Cobb angle (degrees) & 6.3 & 6.3 & 0.796 \\
\hline C2-7 SVA (cm) & 4.4 & 4.2 & 0.586 \\
\hline C1-implant distance (cm) & 0.73 & 0.71 & 0.962 \\
\hline C2 screw type & & & 0.263 \\
\hline Pedicle screw & 18 & 6 & \\
\hline Pars screw & 22 & 2 & \\
\hline Combination or others & 9 & 3 & \\
\hline
\end{tabular}

NDI, neck disability index; SVA, sagittal vertical axis.

fusions. We hypothesized that three risk factors might be associated with postoperative severe headache. First, adults with positive C2-7 SVA or cervical malalignment might increase $\mathrm{C} 1-\mathrm{C} 2$ lordosis in an effort to maintain horizontal gaze. Subsequently, the $\mathrm{C} 2$ nerve root would be impinged due to the hyperlordosis of $\mathrm{C} 1$, and this could cause $\mathrm{C} 2$ nerve irritation and severe headache. Secondly, if the patient had a longer C2-7 SVA, the tonus of the neck muscles could remain under considerable and prolonged tension, which could cause severe headache. Thirdly, a proximal implant such as a rod or screw head can irritate the bony structure or C2 nerve root and cause severe headache. To evaluate the hypothesis and risk factors, we evaluated clinical and radiological findings including T1 slope angle, $\mathrm{C} 1-\mathrm{C} 2$ lordosis angle, $\mathrm{C} 2-7 \mathrm{Cobb}$ angle, C2-7 SVA and C1-implant distance. However, these radiological spinal parameters were not significantly associated with severe headache after PSF from C2 distally. 
Among all patients, 20 patients still had severe headache after this procedure. Of note, 11 patients (10\%) among 60 patients who had preoperative milder headache presented with newly occurring severe headache after surgery. In addition, cervical PSF treated cervicogenic severe headache effectively in 13 patients among the 22 patients who had preoperative severe headache.

The reason for the new prevalence of severe headache following cervical PSF is still unknown. Although axial symptoms (severe neck pain) after cervical laminoplasty have been reported in the literature [4-6], there has been no report on severe headache occurring after cervical posterior surgery. In recent years, a few studies reported axial symptoms occurring after cervical laminectomy with fixation [7,8]. Zhang et al. [7] reported that after laminectomy and lateral mass screw fixation, $40 \%$ of the patients showed axial symptoms postoperatively. This study showed that axial symptom severity correlated positively with the rate of muscle atrophy. Du et al. [8] reported the incidence of axial symptoms at $37.5 \%$ after laminectomy with lateral mass screw fixation, and loss of lordosis was positively related to axial symptom severity. They concluded that axial symptoms were the result of a complication induced by multiple factors and mechanisms after posterior cervical surgery and the explicit pathogenesis required further investigation. Some authors have reported that axial pain after cervical laminoplasty occurred due to neck muscle disruption, especially because of detachment of muscle insertions from the $\mathrm{C} 2$ or $\mathrm{C} 7$ spinous processes, or deep extensor muscles [9-11]. As to the cause of the occurrence of severe headache after PSF C2 distally, in the similar way we considered it to be due to the removal of the muscles attached from $\mathrm{C} 2$ spinous process to allow the insertion of the screw.

On the other hand, Tang et al. [3] used radiological parameters such as $\mathrm{C} 2-7$ lordosis angle and C2-7 SVA to evaluate the relationship between regional cervical sagittal alignment and postoperative outcomes for patients who received multilevel posterior cervical fusion. They demonstrated that the severity of neck pain and disability increases with positive sagittal malalignment following surgical reconstruction. Contrary to their results and the first part of our hypothesis, in our present study, there were no significant associations with between radiological parameters such as cervical lordosis and C2-7 SVA and severe headache. This suggests that there was no C2 impingement around the C1/2 area due to the hyperlor- dosis of $\mathrm{C} 1 / 2$ and that greater C2-7 SVA distance did not increase pressure from the muscles around the neck and skull. Various factors such as revision surgery and a preoperative higher NDI score (neck pain and headache) had significant effects on newly occurring severe headache in this study.

On the other hand, Jansen et al. [12] reported an association between cervicogenic, hemicranial attacks and vascular irritation or compression of the $\mathrm{C} 2$ nerve root. They used electron microscopy to reveal focal, morphological changes of the $\mathrm{C} 2$ nerve root and the ganglion itself. They concluded that these morphological changes were possibly the result of chronic vascular compression. In addition, several authors proposed that one of the most important structures involved in the pathogenesis of occipital headache is the lateral C1-C2 joint [13-15]. Ehni and Benner [13] described a group of elderly patients suffering from an occipital neuralgic pain syndrome aggravated by neck motion due to degenerative changes in a $\mathrm{C} 1-\mathrm{C} 2$ arthrosis.

Several limitations of our study must be acknowledged. First, this was a retrospective study. Second, we did not evaluate the $\mathrm{O}-\mathrm{C} 1$ and $\mathrm{C} 1-2$ joints by computed tomography or magnetic resonance imaging. Severe osteroarthritis of these joints might cause severe headache, and previous literature reported on this in a series of case reports. However, none of those cases demonstrated C1/2 osteoarthritis on plain open-mouth X-ray. Third, we did not perform a C2 nerve block or facet block as a diagnostic tool of severe headache in every case. Thus, we did not determine the actual cause of the severe headache either preoperatively or postoperatively.

However, to the best of our knowledge, this study is the first to report the prevalence of severe headache after PSF from C2 distally and to assess the risk factors for severe headache after PSF from C2 distally through clinical and radiological findings. Thus, the current study should be useful for the spine surgeon making a decision about PSF from C2 distally as an operative treatment for cervical disorders.

\section{Conclusions}

Of 60 patients which had milder headache preoperatively, 11 (18\%) had severe headache after PSF from C2 distally. A higher preoperative NDI score (neck pain and headache) increased the risk for newly occurring severe headache after PSF from C2 distally. 


\section{Conflict of Interest}

No potential conflict of interest relevant to this article was reported.

\section{References}

1. Ahn NU, Ahn UM, Ipsen B, An HS. Mechanical neck pain and cervicogenic headache. Neurosurgery 2007;60(1 Supp1 1):S21-7.

2. Vernon H, Mior S. The Neck Disability Index: a study of reliability and validity. J Manipulative Physiol Ther 1991;14:409-15.

3. Tang JA, Scheer JK, Smith JS, et al. The impact of standing regional cervical sagittal alignment on outcomes in posterior cervical fusion surgery. Neurosurgery 2012;71:662-9.

4. Hosono N, Yonenobu K, Ono K. Neck and shoulder pain after laminoplasty: a noticeable complication. Spine (Phila Pa 1976) 1996;21:1969-73.

5. Kawaguchi $Y$, Matsui H, Ishihara H, Gejo R, Yoshino O. Axial symptoms after en bloc cervical laminoplasty. J Spinal Disord 1999;12:392-5.

6. Yoshida M, Tamaki T, Kawakami M, et al. Does reconstruction of posterior ligamentous complex with extensor musculature decrease axial symptoms after cervical laminoplasty? Spine (Phila Pa 1976) 2002;27: 1414-8.

7. Zhang P, Shen Y, Zhang YZ, Ding WY, Xu JX, Cao JM. Preserving the C7 spinous process in laminectomy combined with lateral mass screw to prevent axial symptom. J Orthop Sci 2011;16:492-7.
8. Du W, Wang L, Shen Y, Zhang Y, Ding W, Ren L. Long-term impacts of different posterior operations on curvature, neurological recovery and axial symptoms for multilevel cervical degenerative myelopathy. Eur Spine J 2013;22:1594-602.

9. Iizuka H, Shimizu T, Tateno K, Toda N, Edakuni H, Shimada H, Takagishi K. Extensor musculature of the cervical spine after laminoplasty: morphologic evaluation by coronal view of the magnetic resonance image. Spine (Phila Pa 1976) 2001;26:2220-6.

10. Shiraishi T, Fukuda K, Yato Y, Nakamura M, Ikegami T. Results of skip laminectomy-minimum 2-year follow-up study compared with open-door laminoplasty. Spine (Phila Pa 1976) 2003;28:2667-72.

11. Takeshita K, Seichi A, Akune T, Kawamura N, Kawaguchi $\mathrm{H}$, Nakamura K. Can laminoplasty maintain the cervical alignment even when the C2 lamina is contained? Spine (Phila Pa 1976) 2005;30:1294-8.

12. Jansen J, Bardosi A, Hildebrandt J, Lucke A. Cervicogenic, hemicranial attacks associated with vascular irritation or compression of the cervical nerve root C2. Clinical manifestations and morphological findings. Pain 1989;39:203-12.

13. Ehni G, Benner B. Occipital neuralgia and the C1-2 arthrosis syndrome. J Neurosurg 1984;61:961-5.

14. Paluzzi A, Belli A, Lafuente J, Wasserberg J. Role of the $\mathrm{C} 2$ articular branches in occipital headache: an anatomical study. Clin Anat 2006;19:497-502.

15. Zhou L, Hud-Shakoor Z, Hennessey C, Ashkenazi A. Upper cervical facet joint and spinal rami blocks for the treatment of cervicogenic headache. Headache 2010;50:657-63. 